\title{
Consequences of eye fluke infection on anti-predator behaviours in invasive round gobies in Kalmar Sound
}

\author{
Henrik Flink $^{1}$ • Jane W. Behrens ${ }^{2} \cdot$ P. Andreas Svensson ${ }^{1} \mathbb{C}$
}

Received: 9 January 2017 / Accepted: 28 March 2017 / Published online: 6 April 2017

(C) The Author(s) 2017. This article is published with open access at Springerlink.com

\begin{abstract}
Larvae of the eye fluke, Diplostomum, emerge from snails and infect fish by penetrating skin or gills, then move to the lens where they may impair the vision of the fish. For the fluke to reproduce, a bird must eat the infected fish, and it has been suggested that they therefore actively manipulate the fish's behaviour to increase the risk of predation. We found that round gobies Neogobius melanostomus, a species that was recently introduced to the Kalmar Sound of the Baltic Sea, had an eye fluke prevalence of 90-100\%. We investigated how the infection related to behavioural variation in round gobies. Our results showed that the more intense the parasiteinduced cataract, the weaker the host's response was to simulated avian attack. The eye flukes did not impair other potentially important anti-predator behaviours, such as shelter use, boldness and the preference for shade. Our results are in accordance with the suggestion that parasites induce changes in host behaviour that will facilitate transfer to their final host.
\end{abstract}

Keywords Biological invasion · Parasitism · PITT .

Trematoda $\cdot$ Anti-predator behaviour

\section{Introduction}

By causing sublethal fitness costs as well as mortality, parasites act as one of the most important selective agents in

P. Andreas Svensson

andreas.svensson@1nu.se

1 Department of Biology and Environmental Science, Linnaeus University, 39182 Kalmar, Sweden

2 National Institute of Aquatic Resources (DTU Aqua), Technical University of Denmark, Charlottenlund, Denmark organisms and shape both evolutionary and ecological processes (Lefèvre et al. 2009; Rohr et al. 2009; SchmidHempel 2009; Kekäläinen et al. 2014). Parasites may also modify host's appearance and manipulate their behaviour (Poulin 1994). Indeed, the behaviour of parasitised animals can be explained as a mixed phenotype, because they often represent a composite of characters originating from both host and parasite genotype (Dawkins 1982; Barber et al. 2000). Expression of mixed phenotypes is particularly common in animals infected by parasites that are transmitted trophically, that is, parasites that require one host to be eaten by another to complete their life cycle (Barber et al. 2000; Poulin 2010). Such parasites can benefit from an increased rate of transmission by manipulating behaviours of their intermediate host so that it becomes more susceptible to predation by the parasite's definitive host (Poulin 1994). It is expected that selection for such host manipulation, referred to as parasite increased trophic transmission (PITT), is strong (Lafferty 1999; Barber et al. 2000; but see Cézilly et al. 2010). If host manipulation results in high probability of predation also by predators not suitable as hosts, the parasite may however fail to improve its transmission rate. This predicts that host manipulation should evolve towards suppression of the risk of being predated by non-hosts relative to host predators (Barber et al. 2000). Despite ample indirect evidence of PITT in a variety of parasitised organisms, there is an on-going debate over how important of a process host manipulation is (e.g. Poulin 1994, 1995, 2010; Cézilly et al. 2010; Hafer and Milinski 2015). In fact, altered behaviours in parasitised animals may rather result from competition between parasite and host interests or they may be a mere side effect from pathology (Poulin 1994, 2010; Barber et al. 2000).

Parasites can play a major role in biological invasions (reviewed in Prenter et al. 2004). For example, exposure to parasites from the newly colonised range can cause 
disadvantages to the invader and may explain spatial and temporal variation in invasion success (Pizzatto et al. 2012). Populations of established introduced species occasionally collapse, known as boom and bust, and such collapses may be ascribed to pathogens or parasites native to the colonised area (Simberloff and Gibbons 2004).

Since 1990, the invasive round goby Neogobius melanostomus (Pallas, 1814), native to the Ponto-Caspian region, has expanded its range and increased in abundance all over the Baltic Sea and the Great Lakes (Sapota and Skóra 2005; Azour et al. 2015; Ojaveer et al. 2015). In the Baltic Sea, it now dominates the catch in various coastal fisheries, and it is frequently found in offshore catches (Ojaveer et al. 2015). Round gobies are like most fishes infected by numerous different parasites both in their native and invasive areas (Kvach et al. 2014). However, to what extent this may affect their behaviour and fitness remains unknown.

The trematode genus Diplostomum contains several common parasites infecting the eyes of freshwater and brackish water fish and thus they are called eye flukes (Chappell 1995; Gibson 1996). The complex life cycle of Diplostomum spp. start with free-swimming miracidiae that in order to reproduce asexually infect aquatic snails (Chappell 1995; Seppälä 2011). Cercariae burst out from snails in multitudes and when they find a fish, their second intermediate host, they infect it through the skin or gills. Within $24 \mathrm{~h}$, the cercariae have migrated to the eye where they develop to metacercariae (Chappell 1995). Eye flukes are most commonly found in fish lenses, but also in the retina or the vitreous humour (Gibson 1996). To reach the next stage in the life cycle, infected fish needs to be eaten by a bird, which often is a gull, tern, goose or duck (Gibson 1996; Seppälä 2011). In the bird intestine, the parasite matures, reproduces sexually and releases its eggs together with the bird faeces.

Eye flukes are known to cause harmful effects to fish as they induce cataract, i.e. lens opacities, by mechanical destruction, metabolic excretions and by being partially or completely opaque (Shariff et al. 1980). High parasite burden in fish may lead to blindness, emaciation and even death (Shariff et al. 1980; Chappell 1995; Karvonen et al. 2004). Recent molecular studies show that it is common for fish to be infected with more than one species of eye fluke, and that different fish species harbour different parasite communities in their lenses (Rellstab et al. 2011). In dace Leuciscus leuciscus (Linnaeus, 1758), three-spined stickleback Gasterosteus aculeatus (Linnaeus, 1758) and Arctic charr Salvelinus alpinus (Linnaeus, 1758), impaired vision due to eye fluke infection has been shown to reduce feeding capability, thereby increase feeding times and predation risk (Crowden and Broom 1980; Owen et al. 1993; Voutilainen et al. 2008). Also, infected dace spend more time near the water surface where they are exposed to avian predation (Crowden and Broom 1980) and infected rainbow trout Oncorhynchus mykiss (Walbaum, 1792) have a reduced escape response towards aerial attacks and thus a suspected increase in susceptibility to predation by birds (Seppälä et al. 2004, 2005b, 2012). How much any of this applies to the round goby remains unknown.

Diplostomum is often the parasite taxa with highest prevalence in round gobies (e.g. Muzzall et al. 1995; Kvach and Skóra 2007; Kvach and Stepien 2008; Francová et al. 2011). However, the prevalence varies considerably, from $0 \%$ in some populations to prevalence's towards $90 \%$ in others (Muzzall et al. 1995; Camp et al. 1999; Kvach et al. 2014). Parasite intensity (i.e. number of eye flukes per infected fish) varies similarly and can locally be very high (Kvach and Skóra 2007; Kvach and Winkler 2011; Kvach et al. 2014). In the present study locations, southeast of Sweden in the Kalmar Sound, the invasive round goby was first observed in 2013 and has since then become one of the most dominant fish species (Nilsson 2014, 2016). Compared to other species of fish, a considerable proportion of round gobies caught at the study location in 2015 and 2016 had intense eye fluke infection (H. Flink and D. Amnebrink, unpublished data). If eye fluke-infected round gobies suffer some of the same effects as described above for other fish hosts, it may affect the potential for the continued invasion of this species. In localities with high infection intensity, such as in the present study location, we hypothesise that eye flukes have harmful effects on their host as seen in other species, for example by impairing vision and thus anti-predator behaviours. We tested this by investigating if the intensity of infection was related to the expression of round goby anti-predator behaviours. Furthermore, we measured prevalence and intensity of eye fluke infections, and carried out molecular species determination of eye flukes.

\section{Method}

\section{Collection and husbandry}

The study was performed at Kalmar Sound Laboratory, Kalmar, Sweden. Two field collections were made using a seine net in shallow water $(0-1 \mathrm{~m})$. In October 2015,88 adult round gobies (average total length \pm S.D. $9.2 \pm 1.5 \mathrm{~cm}$ ) were caught for the behavioural experiments in two bays in Kalmar sound (Kattrumpan 56 39' 56" N, $16^{\circ} 22^{\prime} 25^{\prime \prime}$ E and Tallhagen $56^{\circ} 41^{\prime} 6^{\prime \prime} \mathrm{N}, 16^{\circ} 22^{\prime} 9^{\prime \prime} \mathrm{E}$ ). To get a second estimate of eye fluke prevalence and intensity in the field, 50 more adult round gobies were collected at Kattrumpan in April 2016.

The fish collected in October 2015 were held in stock tanks with continuously flowing brackish water $(7 \pm 0.2 \mathrm{psu})$ until January 2016, 1 month before behavioural experiments began. The fish were then transferred to holding aquaria $(50-60 \mathrm{~L})$ with dechlorinated tap water and commercial sea salt (SERA marine sea salt, Germany) at a salinity of $7.0 \mathrm{psu}$, 
corresponding to the salinity at the location of collection. Four fish were housed in each 40-L aquarium and five fish in each $50-\mathrm{L}$ aquarium. The holding aquaria had individual filters and partial water changes were conducted every second week. All tanks were provided with coarse gravel and plastic tubes $(14 \mathrm{~cm}$ by $\varnothing 4.5 \mathrm{~cm})$ as shelters. The light regime was set to mimic daily changes in the outdoors diurnal light cycle and was changed continuously during the holding period. The water temperature varied between 13 and $15{ }^{\circ} \mathrm{C}$ in a temperature-controlled room. The fish were fed small pieces of herring and blue mussels ad lib three times per week. During the time in these tanks, infected fish fed readily of the provided food, they appeared healthy (except for eye fluke-induced cataract) and behaved normally.

\section{Eye dissection}

Directly following the behavioural trials (October 2015 sample) or maximally 1 week after capture (April 2016 sample), individual fish was euthanised using an overdose of benzocaine. Total length and wet weight was measured to the closest millimetre and milligram, respectively. The cataract score (degree of parasite-induced cataract coverage) was assessed in the fish lenses with a direct ophthalmoscope (HEINE Beta 200S LED, USA). Cataract score was determined with a subjective scale as used in earlier studies (Karvonen et al. 2004; Seppälä et al. 2005ab, 2008, 2011, 2012; Karvonen and Seppälä 2008), where $1=$ no cataracts, $2=$ less than $50 \%$ coverage, $3=$ more than $50 \%$ coverage and $4=100 \%$ coverage or completely white lens. Individual cataract intensity was quantified as the average score from both eyes, allowing for comparison with previous studies (e.g. Seppälä et al. 2005b, 2011; Karvonen and Seppälä 2008). Petri dishes and microscope slides were prepared with saline solution, each eye was removed and put on an individual dish, and the lenses were excised and placed on a slide. All metacercariae from each fish, both from the lens and outside the lens, regardless of developmental stage, were counted with a dissecting microscope. The exact site of parasites found outside the lens (i.e. in the vitreous humour or in tissues under the retina) could not be determined. All flukes found were identified as the genus Diplostomum by morphological characters (Key: Gibson 1996). Metacercariae were put aside in saline solution for further morphological and molecular determination.

\section{Molecular analysis of metacercariae}

Metacercariae from 32 randomly selected round gobies (October 2015 sample) were sampled for molecular species determination. Most parasites were found in the lens of the eye and most were translucent; however, there were also some opaque parasites and some that were found in other areas of the eye. Two parasites from each of the 32 fish were selected at random from the lens, and if the fish had parasites from other areas of the eye and/or that were opaque, these were also sampled. In total, 87 metacercariae were sampled: 64 randomly sampled and in addition 23 that were opaque and/or found outside the lens. Before molecular analysis, the metacercariae were fixed in boiling water for $1 \mathrm{~min}$ and individual parasites put on a microscope slide and photographed through a light microscope to enable morphological comparisons. The parasite was placed in ethanol for 2 to 3 weeks prior to DNA extraction.

DNA was extracted from the metacercariae using the QIAamp DNA Mini Kit. PCR (T100 Thermal Cycler Bio-Rad, CA, USA) was used to amplify sequences containing the ribosomal internal transcribed spacer (ITS) region. The PCR reactions contained $5.0 \mu \mathrm{L}$ DNA extraction, $2.5 \mu \mathrm{L}$ PCR buffer $\left(15 \mathrm{mM} \mathrm{MgCl}_{2}\right.$ ), $0.2 \mu \mathrm{L}$ Taq polymerase $(5.0 \mathrm{U} / \mu \mathrm{L}), 0.1 \mu \mathrm{L}$ dNTP $(10 \mathrm{mM})$ and $0.1 \mu \mathrm{L}$ of each primer, in a total volume of $25 \mu \mathrm{L}$. As forward and reverse primers, D1 (Galazzo et al. 2002) that targets the 3 'end of the $18 \mathrm{~S}$ gene and BD2 (Luton et al., 1992) that targets the 5 'end of the $28 \mathrm{~S}$ gene were used, respectively. The PCR configuration was as follows: first, an initial denaturing step at $95{ }^{\circ} \mathrm{C}$ for $5 \mathrm{~min}$, then 35 cycles of denaturation at $95{ }^{\circ} \mathrm{C}$ for $30 \mathrm{~s}$, annealing at $53{ }^{\circ} \mathrm{C}$ for $30 \mathrm{~s}$, elongation at $72{ }^{\circ} \mathrm{C}$ for $60 \mathrm{~s}$ and a final elongation at $72{ }^{\circ} \mathrm{C}$ for $7 \mathrm{~min}$. To verify the PCR-product, samples were run through electrophoresis $(1.6 \%$ agarose gel containing $0.01 \%$ SYBR Safe stain) and visualised with UV light. Due to methodological difficulties, only 42 out of 87 metacercariae were successfully amplified. Successful amplifications were purified by using polyethylene glycol (PEG) and ethanol precipitation, and sent for sequencing to Eurofins Genomics, Ebersberg, Germany. Sanger sequencing was performed with the 3730XL DNA analyser (Applied Biosystems, MA, USA). As forward and reverse sequencing primers, BD1 (Luton et al. 1992) and BD2 were used, respectively. BD1 targets the 3 'end of the $18 \mathrm{~S}$ gene. Out of 42 sequences, only 32 had sufficient quality, therefore the rest was omitted from the analysis.

A molecular phylogenetic analysis was performed on 32 metacercariae sequences collected from 21 round gobies. In addition, 22 ITS1 sequences of Diplostomum spp. were used as reference, and two sequences of the Diplostomidae species Tylodelphys scheuringi (Hughes, 1929) and Alaria taxideae (Swanson and Erickson 1946) were used as out-group (method adapted from Haarder et al. 2013). Sequences were aligned using ClustalW version 2.1 (Larkin et al. 2007) as implemented in Geneious version 9.1.3 (Kearse et al. 2012) and the phylogenetic analysis was conducted in MEGA7 (Kumar et al. 2016). The analysis was run with the maximum likelihood method based on Kimura $2+$ G (Kimura 1980), as 
suggested as the best-fit model in MEGA7, and a bootstrap consensus tree was inferred from 1000 replicates.

\section{Light avoidance}

To shelter during daylight is a typical behaviour of round gobies (Dubs and Corkum 1996; Borcherding et al. 2013; Capelle et al. 2015) and thus we expect healthy gobies to avoid strong light and prefer shaded areas. We investigated the relationship between eye fluke infection and the preference for shade $(N=88)$. The experimental arenas measured $74 \times 52 \mathrm{~cm}$ (length $\times$ width) with a water depth of $10 \mathrm{~cm}$. To limit the fish from burrowing, the arenas had only $1 \mathrm{~cm}$ layer of fine sand. One half of the aquaria were shaded to a greater extent than the other by using 0,1 or 2-layers of window film $25 \%$ visible light transmittance (BILTEMA, Sweden) that were placed on the glass sheets covering the tank (Fig. 1a). The test was carried out at three different light intensity levels. In the high and medium contrast setting, a $250 \mathrm{~W}$ metal halide lamp (OSRAM HQI-E 250W/D, Germany) was placed above the aquaria in addition to the fluorescent lights that were for the low contrast setting. The light difference in illuminance between light and shade was approximately $35 \times$ (high contrast), $10 \times$ (medium contrast) and $3 \times$ (low contrast). Individual round gobies were netted from its holding aquarium and placed on the border between the bright and the dark half 5-6 $\mathrm{h}$ before the trial. IR-sensitive video cameras above the aquaria were used to study the position of the fish. During $2 \mathrm{~h}$, between 8:30 and 10:30 pm, the location of the fish was recorded and the time spent in the dark versus the bright area was quantified to the nearest second. The proportion of time spent in the darker half was then used to estimate the preference for shade.

\section{Shelter use and boldness}

Chasing and catching fish with dip-net is a simplified method of simulating predator attack and has previously been used to evaluate vulnerability to predation, for example in eye flukeinfected rainbow trout (Seppälä et al. 2004, 2005b; Gopko et al. 2015). To determine if the round gobies differed in their ability to find and use shelter, 61 fish from the light avoidance test were also screened for shelter usage and boldness. The same arenas were used as in the light avoidance test, but without the overhead lamp and the glass covers. Two plastic tubes (14 cm by $\varnothing 4.5 \mathrm{~cm}$, same type as in the holding tanks) were added to the longer sides of each aquarium (Fig. 1b). The fish were then slowly but continuously chased with a rectangular dip-net $(16 \mathrm{~cm} \times 13 \mathrm{~cm})$ until it found shelter in one of the tubes. The complete trial was video-recorded and the time taken from the onset of chasing until the fish was completely inside the tube was subsequently quantified to the nearest second and used as an estimate of ability to find shelter.
When the fish had entered the shelter, it was left alone. The time from this point and until the fish had emerged from the tube with its entire body was used as an estimate of boldness, however, allowing the fish a maximum of $30 \mathrm{~min}$ to emerge from the shelter. Boldness is a trait with high repeatability in individual round gobies ( $R=0.77$, Flink and Svensson, unpublished data) and is often evaluated in studies of animal personalities (Sih et al. 2004).

\section{Response to simulated aerial attack}

In order to study how cataract may affect escape response following aerial attack, the fish previous screened for shelter use and boldness $(N=61)$ were exposed to a simulated aerial predator. The fish were placed in a new experimental arena, measuring $100 \times 100 \mathrm{~cm}$ (Fig. 1c). The water depth was $10 \mathrm{~cm}$ and the water was replaced every fourth trial. The bottom of the arena was white and no shelter was provided to create an area perceived as risky in terms of exposure to aerial predation. The fish was put in a transparent vertical cylinder $(\varnothing 15 \mathrm{~cm})$ in the centre of the aquarium. After giving the fish $10 \mathrm{~min}$ to settle, the cylinder was gently lifted out of the way with a pulley system. Five seconds later, a black square $(25 \times 22 \mathrm{~cm})$ was released from above the fish and fell $78 \mathrm{~cm}$ before stopping immediately above the fish, $1 \mathrm{~cm}$ above the water surface. The escape response was observed and was quantified as either $0=$ no response/freezing, or $1=$ immediate escape (i.e. dashing away from the centre). Other possible escape responses such as slow swimming, jumps and staggered dash (Barber et al. 2004) were not observed.

\section{Data analysis}

The collected data were analysed with $\mathrm{R}$ version 3.2.2 ( $\mathrm{R}$ Core Team 2015). If necessary to obtain normality of residuals, variables were log-transformed, and if parametric assumptions still were not satisfied, non-parametric tests were used. Binary response variables were analysed with generalised linear models (GLM) and depending on the degree of overdispersion, either binomial or quasibinomial errors were used. Boldness, measured as in time to emerge from shelter, was analysed with time-toevent analysis using a Cox proportional hazards regression model in the Survival package (Thernau 2013). This allowed us to account for censoring in the data (i.e. not all fish emerged during the trial). Body condition was calculated by taking the residuals from a mass/length log-log linear regression of all fish caught at the same time (October 2015 sample: $N=88, R^{2}=0.98$; April 2016 sample: $N=50, R^{2}=0.98$ ). When calculating the total number of metacercariae per fish, the sum of parasites in both eyes was used. However, in four fish, the total number of parasites was extrapolated from one eye due to unsuccessful quantification of metacercariae in the other eye. In the analysis of behavioural experiments we omitted, two fish with both their lenses erupted. 


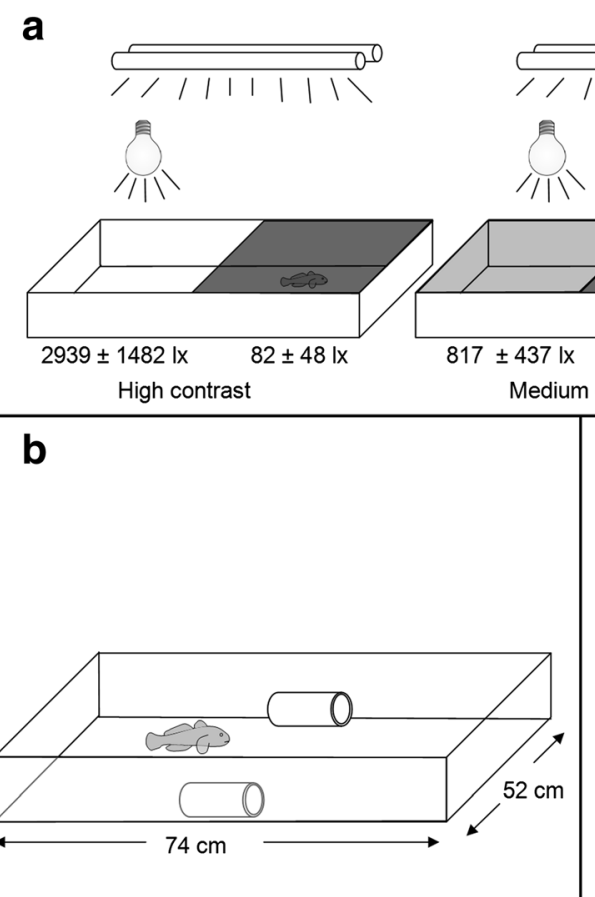

Fig. 1 Test arenas used to quantify anti-predator behaviours in round gobies. In the light avoidance test (a), three different contrast settings were created using combinations of overhead fluorescent lights and shade films. An additional light was placed above the brighter part of the arena in the high and medium contrast levels. Numbers indicate mean illuminance in lux \pm S.D. In the sheltering and boldness test (b), two shelters

\section{Results}

\section{Assessment of cataract and dissection}

The prevalence of Diplostomum spp. in round gobies collected in October 2015 was $98 \%$ ( 86 infected fish out of 88). Two fish did not have any eye flukes but had erupted lenses on both eyes. Because it is known that severe infections may lead to destruction of the lenses (Shariff et al. 1980), it is likely that these fish had been infected earlier. One more fish had an erupted lens, while the other lens was intact but with a high intensity of eye flukes. All fish had detectable eye flukeinduced cataract, and in $35 \%$ of fish, the cataract coverage was more than $50 \%$ in both eyes (mean cataract score $\geq 3$, Fig. 2). The average intensity ( \pm standard deviation) of metacercariae per fish was $58 \pm 39(N=86)$. As expected, fish with higher parasite load had higher cataract score (Spearman's rank correlation: $N=55, \rho=0.43, p<0.001$ ). However, there was no correlation between the total amount of eye flukes and the condition of the fish (Spearman's rank correlation: $N=86, \rho=0.14, p=0.20$, Fig. 3), or between cataract score and fish condition (Spearman's rank correlation: $N=56, \rho=0.16, p=0.24)$. It should be noted that these fish had been fed ad lib for 4 months in the lab prior to experiments. Larger fish had higher load of eye flukes (Log-log linear regression model, $\left.N=86, R^{2}=0.27, p<0.001\right)$. In total, 4722 eye flukes were found and according to their morphology, all flukes were fully developed metacercariae and infective for birds (see Sweeting 1974). Seven metacercariae were found outside the lens in six different fish.

A second sample $(N=50)$ was collected in April 2016 in order to compare prevalence between newly caught fish with that of the lab-housed fish. Prevalence in this sample was $90 \%$, which is not significantly different from the first sample (Fisher's exact test, $p=0.80)$. However, the mean intensity, $27 \pm 30(N=45)$, was lower than in the first collection (Wilcoxon rank sum test $W=882.5, p<0.001)$. There was no correlation between the length of the fish and the amount of eye flukes (Spearman's rank correlation $N=50, \rho=0.23, p=0.12$ ). There was however a negative association between the parasite load and the condition of the fish (Spearman's rank correlation $N=50, \rho=0.59$, $p<0.001$, Fig. 3). In the second sample, a total of 1201 eye flukes were found. A large number of these were immature. Only one eye fluke was found outside the lens.

\section{Molecular analysis of metacercariae}

The phylogenetic analysis branched the isolated parasites into three different clades (Fig. 4). In total, 19 isolates were determined as Diplostomum mergi (Dubois, 1932), 12 as 
Fig. 2 Infected round gobies from the October 2015 sample. Cataract intensity was assessed with an ophthalmoscope. Most commonly, fish had less than $50 \%$ cataract coverage (a); however, several fish had more than $50 \%$ coverage $(\mathbf{b}, \mathbf{c})$. A few fish had $100 \%$ coverage and in rare cases completely white lenses, sometimes dislocated $(\mathbf{d})$ or completely erupted

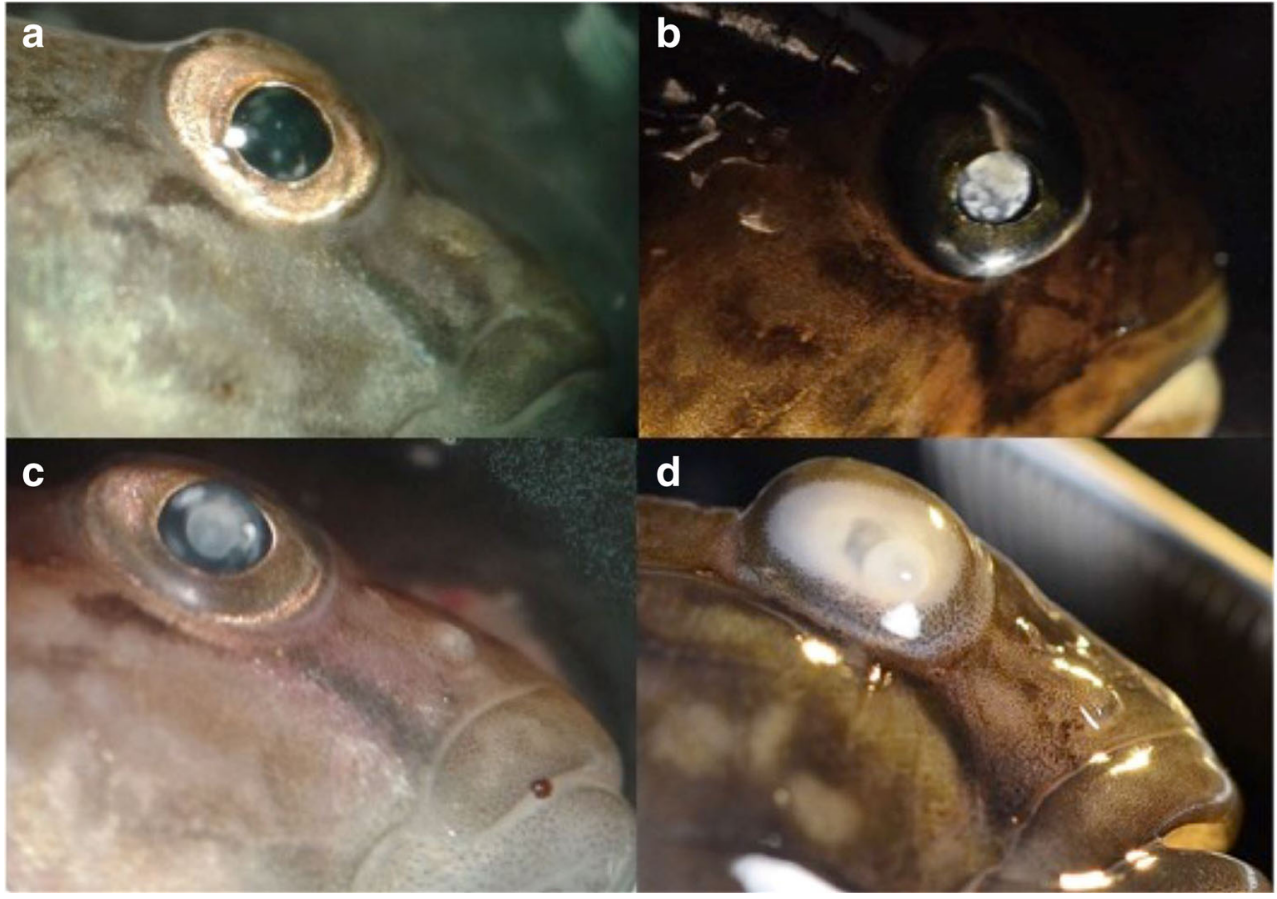

Diplostomum paracaudum (Iles, 1959) and one as Diplostomum baeri (Dubois, 1937) (see Fig. 5 for photographs). The $D$. baeri isolate was found outside the lens and was opaque. One isolate of $D$. mergi and one of $D$. paracaudum were also found outside the lens, whilst the others were found in the lens. In nine fish, successful sequencing was performed from more than one parasite. Out of these, five fish were infected with both $D$. mergi and $D$. paracaudum.

\section{Light avoidance}

The majority of fish were actively swimming during observations, and 71 out of 78 fish spent more than $50 \%$ of the time in the dark half (Wilcoxon signed rank test $V=46, p<0.001$, Fig. 6). This was true for all three settings, although in the lowest contrast setting fish spent relatively more time in the brighter area (Kruskal-Wallis rank sum test $\chi^{2}=8.91$, $p=0.01$ ). Cataract score was not correlated to the preference for shade in either fish from high (Spearman's rank correlation $N=17, \rho=0.24, p=0.36$ ) or medium contrast treatment (Spearman's rank correlation $N=32, \rho=0.14, p=0.43$ ). Cataract score was not assessed in fish from low contrast trials; however, the highly correlated measure parasite intensity was unrelated to shade preference (Spearman's rank correlation $N=26, \rho=0.04, p=0.84$ ).

\section{Shelter use and boldness}

All the tested fish found shelter with a maximum time of $102 \mathrm{~s}$ (mean $26 \pm 23$ ). There was no significant relationship between cataract score and the time it took to reach shelter (GLM with Gamma errors $N=53$, $t=1.18, p=0.25)$. The fish that did not emerge from the shelter within the maximum time ( 23 of 57 fish) obtained the ceiling value of $1800 \mathrm{~s}(30 \mathrm{~min})$ and were treated as censored data points in this analysis. The average time the fish took to emerge from shelter was $1197 \pm 608$ s. Boldness was not associated with cataract score (Cox proportional hazards regression model $N=54$,

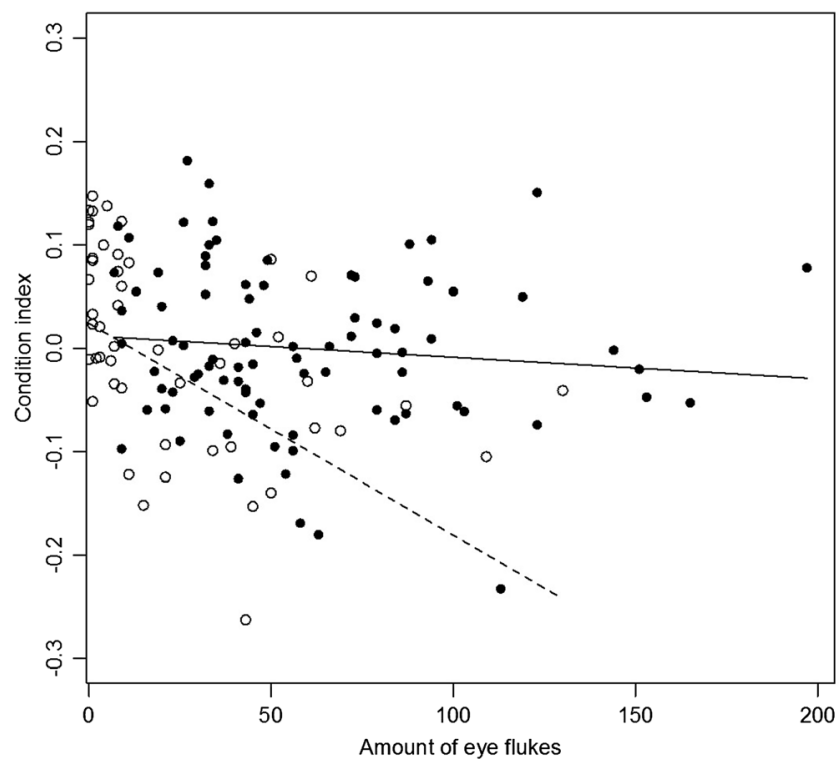

Fig. 3 The body condition of fish with high parasite load was lower than average in field (April 2016 sample: white dots, dashed line). There was no such correlation in fish that had been held in lab for 4 months (October 2015 sample: black dots, solid line) 
Fig. 4 Molecular phylogenetic tree estimated with the maximum likelihood method based on the Kimura 2-parameter model of 54 sequences of Diplostomum spp. and two out-group sequences. Sequences of the 32 parasites isolated from round gobies (October 2015 sample) are emphasised in bold. The other 22 sequences are representatives for known Diplostomidae species (see Haarder et al. 2013 for selection criteria). The analysis shows that the eye flukes sampled in this study are branched in three clades. Bootstrap values above $50 \%$ are shown next to each branch. The tree is drawn in scale, with branch lengths measured in the number of substitutions per site

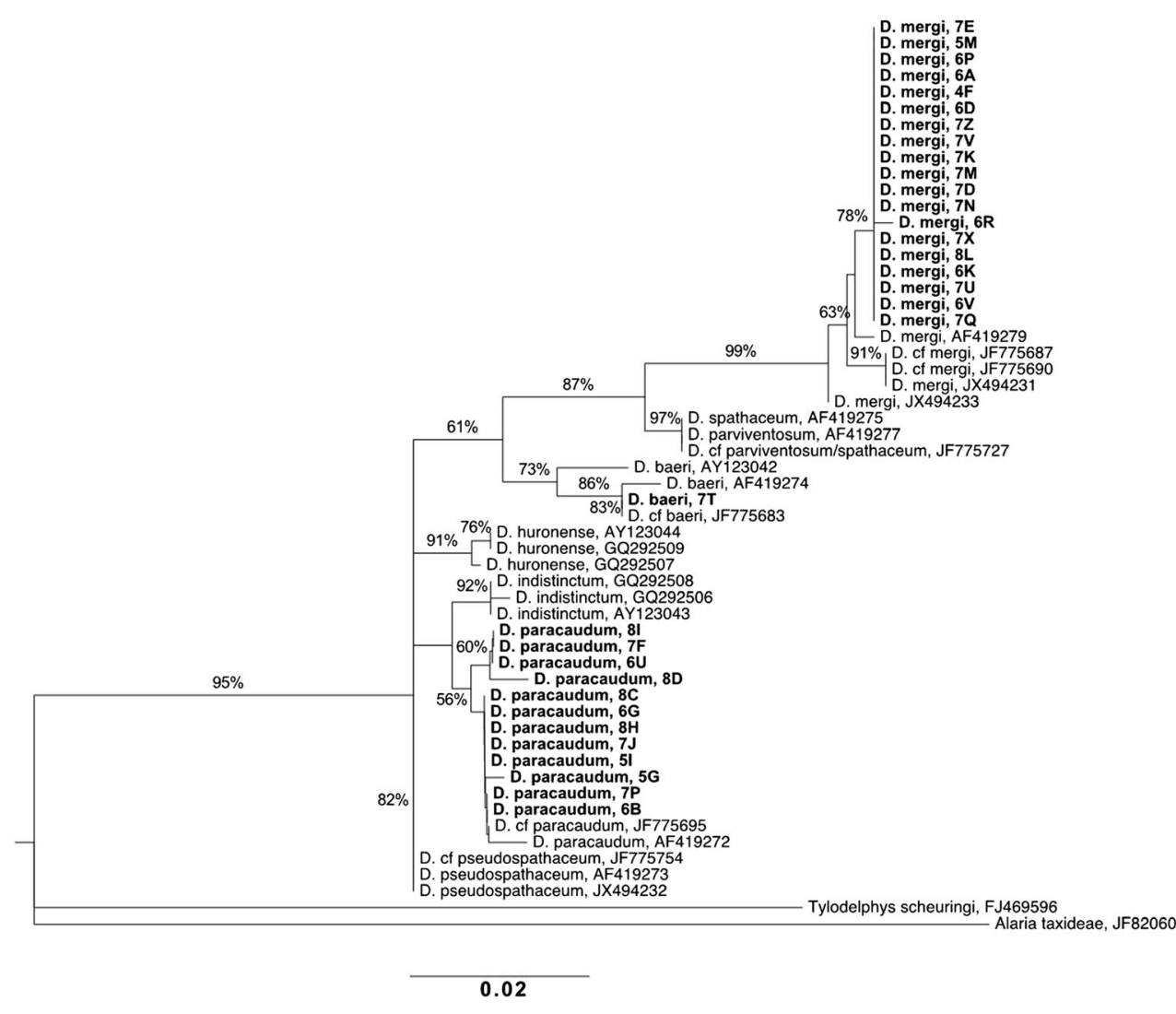

shelter (Cox proportional hazards regression model $N=56, \mathrm{z}=1.07, p=0.29)$. $z=1.04, p=0.30)$. There was no association between the time it took to find shelter and the time to emerge from
Fig. 5 A selection of metacercariae from round goby lenses, determined by phylogenetic analysis as $D$. mergi $(\mathbf{a}-\mathbf{c})$ and $D$. paracaudum $(\mathbf{d}-\mathbf{f})$. Also, one specimen of D. baeri (g) found outside the lens. Parasites were fixed in boiling water and then photographed through a light microscope. Scale bars $0.1 \mathrm{~mm}$
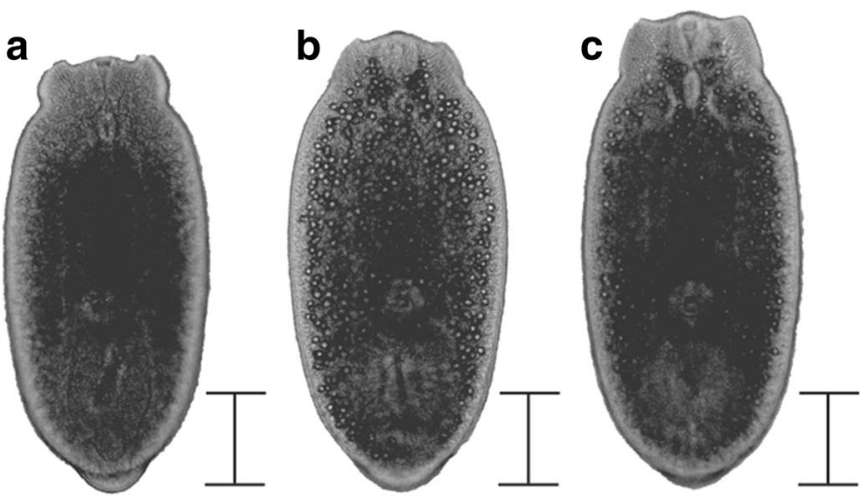

d

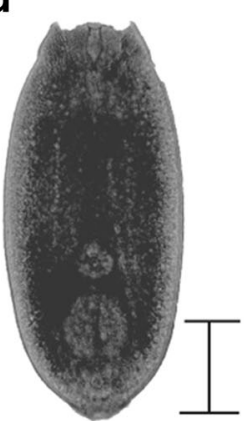

e

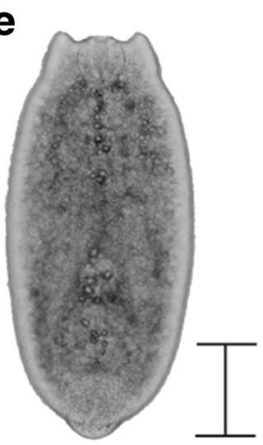

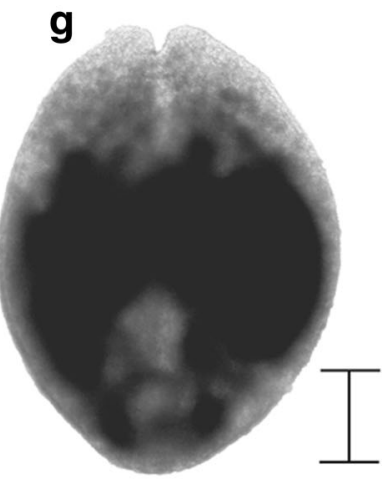




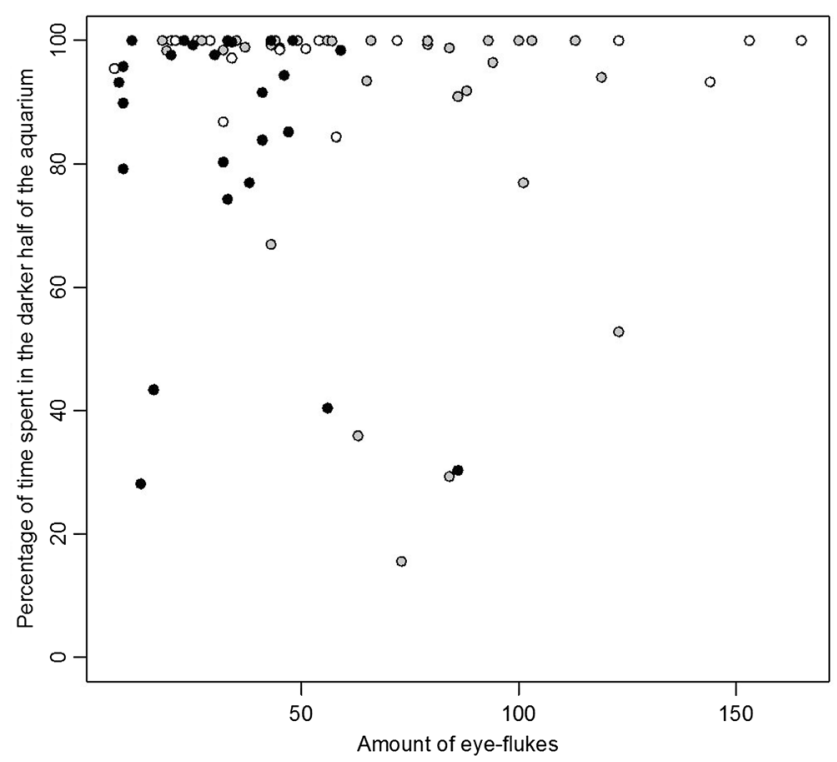

Fig. 6 Percentage of time spent in the darker half of the aquarium during the three light intensity treatments in relation to the amount of eye flukes the round gobies harboured. Independent of treatment, the fish spent most of the time in the darker half. The different light intensity treatments are indicated as circles with white (high contrast), grey (medium contrast) and black background (low contrast)

\section{Response to simulated aerial attack}

The cataract score was negatively related to the propensity to escape following the simulated attack, that is, fish with severe cataract fled less frequently (GLM with quasibinomial errors $N=53, t=2.50, p=0.01$, Fig. 7). None of the fish with $>50 \%$ cataract coverage in both eyes (cataract score $\geq 3$ ) fled.

\section{Discussion}

Previous studies have proposed that eye flukes induce phenotypic changes that increase the susceptibility of fish hosts to predation (Crowden and Broom 1980; Owen et al. 1993; Seppälä et al. 2004, 2005a, b, 2008; Voutilainen et al. 2008) and that this effect is caused by impaired vision of the fish (Seppälä et al. 2005b, 2012). It has also been proposed that the increased susceptibility to predation is specifically targeting avian predators, suggesting it is an adaptation to increase the likelihood of the suitable final host eating the fish (Seppälä et al. 2006a, 2012). Our results are in accordance with these notions, suggesting that round gobies with severe eye fluke infection have reduced escape response to simulated aerial attack while more general anti-predator behaviours appear unaffected.

Fish clearly avoided the brighter parts of the aquarium irrespectively of degree of cataract. Such a preference was expected from round gobies if they perceive the darker part as less risky in terms of exposure to visual predators, and, clearly, the parasite did not change the individual fish's ability to respond to

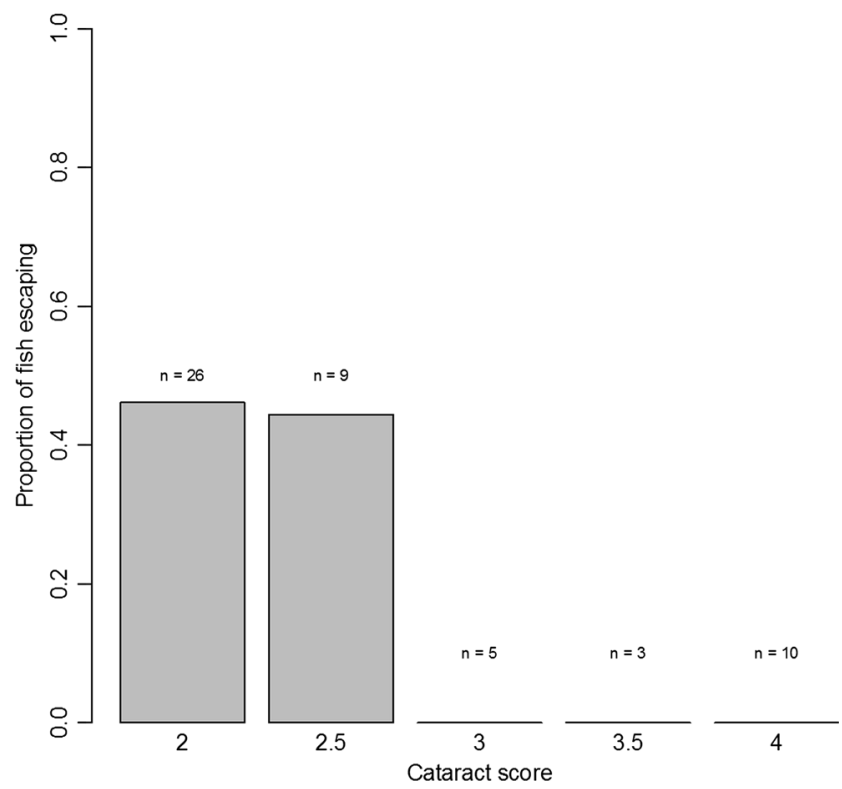

Fig. 7 Round gobies were less likely to flee following a simulated aerial attack when cataract coverage was severe. No fish with more than $50 \%$ cataract coverage in both eyes responded by fleeing. The cataract score (average from both eyes) was calculated as follows: $1=$ no cataracts, $2=$ less than $50 \%$ coverage, $3=$ more than $50 \%$ coverage and $4=100 \%$ coverage or completely white lens

differences in light intensity. Eye fluke-induced cataract has been suggested to impair nocturnal behaviour of Arctic charr, increasing their susceptibility to visual predators (Voutilainen et al. 2008). In addition, rainbow trout with cataract has reduced preference of dark background colouration, where the fish are less conspicuous, and impaired ability to adjust colouration to the background (Seppälä et al. 2005a). Our results demonstrate that round gobies with intense cataract could assess the present light intensity differences, including a relatively low contrast. However, it remains unknown whether round goby nocturnal behaviours are affected by cataract. The severity of the parasiteinduced cataract was neither associated with the individuals' ability to find shelter when chased nor with their boldness. This is in agreement with a study on rainbow trout where time spent in shelter, general activity level, and reaction to a simulated fish attack was unaffected by fluke infection and cataract (Seppälä et al. 2012). They suggested that host manipulation by eye flukes is driven by impaired vision and that fish vision is crucial to detect and avoid aerial predators, whereas eye fluke infection has no or little effect on other senses, such as the lateral line, which can be used to avoid for example predatory fish (Seppälä et al. 2012).

The observed reduced escape response following an aerial attack in fish with severe cataract suggests that this behavioural change is indeed caused by parasites, and likely by impairing the vision. This is in agreement with previous results in rainbow trout, where infected fish exhibit reduced escape response to aerial attacks (Seppälä et al. 2004, 2005b). However, as also shown by Seppälä et al. (2005b), 
the effect was observable first when cataract coverage had exceeded $50 \%$. Thus, when cataract formation is intense, eye fluke infection appears to change host escape response in a way that increases susceptibility to predatory birds (Seppälä et al. 2004, 2005b, 2008). Yet, despite the consensus between these laboratory-derived results, the only available field study found that susceptibility to bird predation was not affected by eye flukes in rainbow trout (Seppälä et al. 2006b).

The molecular species determination showed that the parasite isolates from infected round gobies in the Kalmar Sound branched within clades of three different Diplostomum species, namely D. mergi, D. paracaudum and D. baeri. However, the small sample size may have led to an underestimation of the actual number of Diplostomum species. Eye fluke species typically have a low specificity in what fish species they infect as intermediate host (Karvonen et al. 2006) and fish are often infected by multiple fluke species at the same time (Rellstab et al. 2011 and references therein). Our molecular analysis suggests that $D$. mergi and $D$. paracaudum often coinfects round gobies. All three species are well known to the Baltic region (Rellstab et al. 2011), thus it is unlikely that these parasites were introduced together with the round goby when it first was transported to the Baltic Sea. D. baeri is known to reside in the vitreous humour of the eye (Rellstab et al. 2011), and the only isolate we found of this species was situated outside the lens. The molecular species determination is not absolutely certain without other kinds of data, such as morphometric measurements, and should be interpreted with caution (Cavaleiro et al. 2012).

The field prevalence of eye flukes in the round gobies caught in Kalmar Sound in this study were remarkably high at $90-100 \%$, yet still comparable to prevalence's reported in round gobies from both the Great Lakes of North America (Muzzall et al. 1995) and the Dnieper delta in the Black Sea, Ukraine (Kvach et al. 2014). Eye fluke prevalence and intensity are known to change seasonally, with the highest levels during the warmer part of the year (Mehrdana et al. 2015), and this may be one explanation for the small but significant difference in intensity between our two sampling occasions. It is common that Diplostomum prevalence and intensity can become very high, at least locally. From the Bothnian bay, in the Baltic Sea, prevalences of $90-100 \%$ and intensities above 20 parasites per infected individual have been reported in several other fish species, such as dace, roach Rutilus rutilus (Linnaeus, 1758), and ruffe Gymnocephalus cernua (Linnaeus, 1758) (Seppälä et al. 2011). All fish caught in the October sample had detectable eye fluke-induced cataract and one third of the fish had more than $50 \%$ cataract coverage in both eyes. If these numbers are representative for the Kalmar Sound population, a large proportion of the fish would potentially have an increased susceptibility to predation by piscivorous birds. There may however be differences in eye fluke prevalence and intensity between different depths and habitats of Kalmar Sound and it is possible that the proportion of fish with severe cataract may be selectively removed by predators.

Only in the first sample did we find a correlation between the length of the fish and the amount of eye flukes. We expected this since infection of eye flukes is cumulative through the life of fish and longer fish are expected to be older (Wootten 1974; Owen et al. 1993). In the second sample, the fish were smaller and varied less in length, and there may thus have been insufficient variation for this effect to be detected. In contrast, a negative correlation between the eye fluke intensity and the condition of the fish was only found in the second sample, where fish were dissected shortly after capture. Since this relationship was not found in the fish that had been fed ad libitum for 4 months in the lab, a possible explanation is that infected fish in the field have an impaired feeding due to eye fluke-induced cataract, as observed in several other fish species (Crowden and Broom 1980; Owen et al. 1993; Voutilainen et al. 2008).

The most important avian predators of round gobies in the Baltic Sea are great cormorants Phalacrocorax carbo (Linnaeus, 1758) and grey herons Ardea cinerea (Linnaeus, 1758), and they may locally feed almost exclusively on round gobies (Jakubas 2004; Bzoma and Meissner 2005). Therefore, it has been suggested that these species can help reduce the population growth of the round gobies in the Baltic (Ojaveer et al. 2015). It is plausible that the impaired escape response of infected round gobies to aerial attacks observed in this study might increase susceptibility to predation by the great heron and other birds that feed by striking from above. Intense eye fluke infection may thus, potentially, help limit the severity of the ongoing round goby invasion of the Baltic Sea.

In conclusion, our results show that eye fluke infection is highly prevalent in round gobies from the present study location in the Baltic Sea, and that several native fluke species infect round gobies simultaneously. Our results also suggest that eye fluke infection causes no changes in shelter behaviour, boldness or preference for shade. However, intense parasite-induced cataract impairs the escape response to aerial attacks, which might increase susceptibility to bird predation. Intense infection is also correlated with poor condition of fish in field, possibly due to impaired feeding capability.

Acknowledgements We are grateful to H. Johansson, D. Amnebrink, K. Baskaran, K. Tsimpos, O. Nordahl, P. Koch-Schmidt and E. Fridolfsson for help in the field and laboratory. We thank C. Bunse and A. Forsman for valuable comments on the manuscript. Material support was received from J. Waldenström and the Zoonotic Ecology and Epidemiology group of Linnaeus University.

\section{Compliance with ethical standards}

Ethical approval All applicable international, national and/or institutional guidelines for the care and use of animals were followed. Animal trials were approved by Linköping Animals Experiment Ethics Board (d/nr 93-15). 
Open Access This article is distributed under the terms of the Creative Commons Attribution 4.0 International License (http:// creativecommons.org/licenses/by/4.0/), which permits unrestricted use, distribution, and reproduction in any medium, provided you give appropriate credit to the original author(s) and the source, provide a link to the Creative Commons license, and indicate if changes were made.

\section{References}

Azour F, van Deurs M, Behrens J, Carl H, Hüssy K, Greisen K, Ebert R, Møller PR (2015) Invasion rate and population characteristics of the round goby Neogobius melanostomus: effects of density and invasion history. Aquat Biol 24:41-52. doi:10.3354/ab00634

Barber I, Hoare D, Krause J (2000) Effects of parasites on fish behaviour: a review and evolutionary perspective. Rev Fish Biol Fisher 10: 131-165. doi:10.1023/A:1016658224470

Barber I, Walker P, Svensson PA (2004) Behavioural responses to simulated avian predation in female three spined sticklebacks: the effect of experimental Schistocepthalus solidus infection. Behaviour 141: 1425-1440. doi:10.1163/1568539042948231

Borcherding J, Hertel A, Breiden S (2013) Acitivity and competitive behaviour of invasive Neogobius melanostomus and Ponticola kessleri (Gobiidae) from the River Rhine, Germany. Ethol Ecol Evol 25:351-365. doi:10.1080/03949370.2013.806361

Bzoma S, Meissner W (2005) Some results of long-term counts of waterbirds wintering in the western part of the gulf of Gdańsk (Poland), with special emphasis on the increase in the number of cormorants (Phalacrocorax carbo). Acta Zool Lit 15:105-108. doi:10.1080/ 13921657.2005.10512383

Camp JW, Blaney LM, Barnes DK (1999) Helminths of the round goby, Neogobius melanostomus (Perciformes: Gobiidae), from southern Lake Michigan, Indiana. J Helminthol Soc Wash 66:70-72

Capelle PM, McCallum ES, Balshine S (2015) Aggression and sociality: conflicting or complementary traits of a successful invader? Behaviour 152:127-146. doi:10.1163/1568539X-00003235

Cavaleiro FI, Pina S, Russell-Pinto F, Rodrigues P, Formigo NE, Gibson DI, Santos MJ (2012) Morphology, ultrastructure, genetics, and morphometrics of Diplostomum sp. (Digenea: Diplostomidae) metacercariae infecting the European flounder, Platichthys flesus (L.) (Teleostei: Pleuronectidae), off the northwest coast of Portugal. Parasitol Res 110:81-93. doi:10.1007/s00436-011-2453-x

Cézilly F, Thomas F, Médoc V, Perrot-Minnot M-J (2010) Hostmanipulation by parasites with complex life cycles: adaptive or not? Trends Parasitol 26:311-317. doi:10.1016/j.pt.2010.03.009

Chappell LH (1995) The biology of diplostomatid eyeflukes of fishes. J Helminthol 69:97-101. doi:10.1017/S0022149X00013961

Crowden AE, Broom DM (1980) Effects of the eyefluke, Diplostomum spathaceum, on the behaviour of dace (Leuciscus leuciscus). Anim Behav 28:287-294. doi:10.1016/S0003-3472(80)80031-5

Dawkins R (1982) The extended phenotype. Oxford University Press, New York

Dubs DOL, Corkum LD (1996) Behavioral interactions between round gobies (Neogobius melanostomums) and mottled sculpins (Cottus bairdi). J Great Lakes Res 22:838-844. doi:10.1016/S03801330(96)71005-5

Francová K, Ondračková M, Polačik M, Jurajda P (2011) Parasite fauna of native and non-native populations of Neogobius melanostomus (Pallas, 1814) (Gobiidae) in the longitudinal profile of the Danube River. J Appl Ichtyol 27:879-886. doi:10.1111/j.1439-0426.2010. 01582.x

Galazzo DE, Dayanandan S, Marcogliese DJ, McLaughlin JD (2002) Molecular systematics of some North American species of
Diplostomum (Digenea) based on rDNA-sequence data and comparisons with European congeners. Can J Zool 80:2207-2217. doi:10. 1139/Z02-198

Gibson DI (1996) Trematoda. In: Margolis L, Kabata Z (eds) Guide to the parasites of fishes of Canada, part IV. NRC Press, Ottawa, pp 1-373

Gopko M, Mikheev VN, Taskinen J (2015) Changes in host behaviour caused by immature larvae of the eye fluke: evidence supporting the predation suppression hypothesis. Behav Ecol Sociobiol 69:17231730. doi:10.1007/s00265-015-1984-z

Haarder S, Jørgensen K, Kania PW, Skovgaard A, Buchmann K (2013) Occurrence of Diplostomum pseudospathaceum Niewiadomska, 1984 and D. mergi Dubois, 1932 (Digenea: Diplostomidae) in Danish freshwater snails: ecological and molecular data. Folia Parasitol 60:177-180. doi:10.14411/fp.2013.020

Hafer N, Milinski M (2015) An experimental conflict of interest between parasites reveals the mechanism of host manipulation. Behav Ecol 27:617-627. doi:10.1093/beheco/arv200

Jakubas D (2004) The response of the grey heron to a rapid increase of the round goby. Waterbirds 27:304-307. doi:10.1675/1524-4695(2004) 027[0304:TROTGH]2.0.CO;2

Karvonen A, Seppälä O (2008) Eye fluke infection and lens size reduction in fish: a quantitative analysis. Dis Aquat Org 80:21-26. doi:10. 3354/dao01918

Karvonen A, Seppälä O, Valtonen ET (2004) Eye fluke-induced cataract formation in fish: quantitative analysis using an ophthalmological microscope. Parasitology 129:473-478. doi:10.1017/ S0031182004006006

Karvonen A, Terho P, Seppälä O, Jokela J, Valtonen ET (2006) Ecological divergence of closely related Diplostomum (Trematoda) parasites. Parasitology 133:229-235. doi:10.1017/ S0031182006000242

Kearse M, Moir R, Wilson A, Stones-Havas S, Cheung M, Sturrock S, Buxton S, Cooper A, Markowitz S, Duran C, Thierer T, Ashton B, Mentjies P, Drummond A (2012) Geneious basic: an integrated and extendable desktop software platform for the organization and analysis of sequence data. Bioinformatics 28:1647-1649. doi:10.1093/ bioinformatics/bts199

Kekäläinen J, Lai Y-T, Vainikka A, Sirkka I, Kortet R (2014) Do brain parasites alter host personality? Experimental study in minnows. Behav Ecol Sociobiol 68:197-204. doi:10.1007/s00265-013-16342

Kimura M (1980) A simple method for estimating evolutionary rate of base substitutions through comparative studies of nucleotide sequences. J Mol Evol 16:111-120. doi:10.1007/BF01731581

Kumar S, Stecher G, Tamura K (2016) MEGA7: molecular evolutionary genetics analysis version 7.0 for bigger datasets. Mol Biol Evol. doi: 10.1093/molbev/msw054

Kvach Y, Kornyychuk Y, Mierzejewska K, Rubtsova N, Yurakhno V, Grabowska J, Ovcharenko M (2014) Parasitization of invasive gobiids in the eastern part of the central trans-European corridor of invasion of Ponto-Caspian hydrobionts. Parasitol Res 113:16051624. doi:10.1007/s00436-014-3791-2

Kvach Y, Skóra KE (2007) Metazoa parasites of the invasive round goby Apollonia melanostoma (Neogobius melanostomus) (Pallas) (Gobiidae: Osteichthyes) in the Gulf of Gdańsk, Baltic Sea, Poland: a comparison with the Black Sea. Parasitol Res 100:767774. doi:10.1007/s00436-006-0311-z

Kvach Y, Stepien CA (2008) Metazoan parasites of introduced round and tubenose gobies in the Great Lakes: support for the "enemy release hypothesis". J Great Lakes Res 34:23-35. doi:10.3394/03801330(2008)34[23:MPOIRA]2.0.CO;2

Kvach Y, Winkler HM (2011) The colonization of the invasive round goby Neogobius melanostomus by parasites in new localities in the southwestern Baltic Sea. Parasitol Res 109:769-780. doi:10. 1007/s00436-011-2321-8 
Lafferty KD (1999) The evolution of trophic transmission. Parasitol Today 15:111-115. doi:10.1016/S0169-4758(99)01397-6

Larkin MA, Blackshields G, Brown NP, Chenna R, McGettigan PA, McWilliam H, Valentin F, Wallace IM, Wilm A, Lopez R, Thompson JD, Gibson TJ, Higgins DG (2007) Clustal W and Clustal X version 2. Bioinformatics 23:2947-2948. doi:10.1093/ bioinformatics/btm 404

Lefèvre T, Lebarbenchon C, Gauthier-Clerc M, Missé D, Poulin R, Thomas F (2009) The ecological significance of manipulative parasites. Trends Ecol Evol 24:41-48. doi:10.1016/j.tree.2008.08.007

Luton K, Walker D, Blair D (1992) Comparisons of ribosomal transcribed spacers from two congeneric species of flukes (Platyhelminthes: Trematoda: Digenea). Mol Biochem Parasitol 56:323-328. doi:10. 1016/0166-6851(92)90181-I

Mehrdana F, Marana MH, Skov J, Bahlool QZM, Sindberg D, Mundeling M, Overgaard BC, Kania PW, Buchmann K (2015) Eye fluke infection status in Baltic cod, Gadus morhua, after three decades and their use as ecological indicators. Acta Parasitol 60:423-429. doi:10. 1515/ap-2015-0058

Muzzall PM, Peebles CR, Thomas MV (1995) Parasites of the round goby, Neogobius melanostomus, and tubenose goby, Proterorhinus marmoratos (Perciformes: Gobiidae), from the St. Clair River and Lake St. Clair, Michigan. J Helminthol Soc Wash 62:226-228

Nilsson J (2014) Inventering av det strandnära yngel- och småfisksamhället i Kalmar, Mönsterås och Västervik, juni 2014. Department of Biology and Environmental Science, Linnaues University. Report 2014:6

Nilsson J (2016) Inventering av det strandnära yngel- och småfisksamhället, samt utbredning av svartmunnad smörbult längs Kalmar läns kust, 2016. Department of Biology and Environmental Science, Linnaues University. Report 2016:5

Ojaveer H, Galil BS, Lehtiniemi M, Christoffersen M, Clink S, Florin AB, Gruszka P, Puntila R, Behrens JW (2015) Twenty five years of invasion: management of the round goby Neogobius melanostomus in the Baltic Sea. Manag Biol Invasion 6:329-339. doi:10.3391/ mbi.2015.6.4.02

Owen SF, Barber I, Hart PJB (1993) Low level infection by eye fluke, Diplostomum spp., affects the vision of three-spined sticklebacks, Gasterosteus aculeatus. J Fish Biol 42:803-806. doi:10.1111/j. 1095-8649.1993.tb00387.x

Pizzatto L, Kelehear C, Dubey S, Barton D, Shine R (2012) Host-parasite relationships during a biological invasion: 75 year postinvasion, cane toads and sympatric Australian frogs retain separate lungworm faunas. J Wildlife Dis 48:951-961. doi:10.7589/2012-02-050

Poulin R (1994) The evolution of parasite manipulation of host behaviour: a theoretical analysis. Parasitology 109:109-118. doi:10.1017/ S0031182000085127

Poulin R (1995) "Adaptive" changes in the behaviour of parasitized animals: a critical review. Int J Parasitol 25:1371-1383. doi:10. 1016/0020-7519(95)00100-X

Poulin R (2010) Parasite manipulation of host behaviour: an update and frequently asked questions. Adv Stud Behav 41:151-186. doi:10. 1016/S0065-3454(10)41005-0

Prenter J, MacNeil C, Dick JT-A, Dunn AM (2004) Roles of parasites in animal invasions. Trends Ecol Evol 19:385-390. doi:10.1016/j.tree. 2004.05.002

Core Team R (2015) R: a language and environment for statistical computing. R: Found Statistical Computing, Vienna, Austria http:// www.r-project.org/

Rellstab C, Louhi KR, Karvonen A, Jokela J (2011) Analysis of trematode parasite communities in fish eye lenses by pyrosequencing of naturally pooled DNA. Infect Genet Evol 11:1276-1286. doi:10. 1016/j.meegid.2011.04.018

Rohr JR, Swan A, Raffel TR, Hudson PJ (2009) Parasites, info-disruption, and the ecology of fear. Oecologia 159:447-454. doi:10.1007/ s00442-008-1208-6

Sapota MR, Skóra KE (2005) Spread of alien (non-indigenous) fish species Neogobius melanostomus in the Guld of Gdansk (south Baltic). Biol Invasions 7:157-164. doi:10.1007/s10530-004-9035-0

Schmid-Hempel P (2009) Parasites - the new frontier: celebrating. Darwin 200. Biol Lett 5:625-627. doi:10.1098/rsbl.2009.0589

Seppälä O (2011) Manipulation of fish phenotype by parasites. In: Farrell AP (ed) Encyclopedia of fish physiology: from genome to environment, vol volume 1. Academic Press, San Diego, pp 777-782

Seppälä O, Karvonen A, Valtonen ET (2004) Parasite-induced change in host behaviour and susceptibility to predation in an eye fluke-fish interaction. Anim Behav 2:257-263. doi:10.1016/j.anbehav.2003. 10.021

Seppälä O, Karvonen A, Valtonen ET (2005a) Impaired crypsis of fish infected with a trophically transmitted parasite. Anim Behav 70: 895-900. doi:10.1016/j.anbehav.2005.01.021

Seppälä O, Karvonen A, Valtonen ET (2005b) Manipulation of fish host by eye flukes in relation to cataract formation and parasite infectivity. Anim Behav 70:889-894. doi:10.1016/j.anbehav.2005.01.020

Seppälä O, Karvonen A, Valtonen ET (2006a) Host manipulation by parasites and risk of non-host predation: is manipulation costly in an eye fluke-fish interaction? Evol Ecol Res 8:871-879. doi:10. 1098/rspb.2008.0152

Seppälä O, Karvonen A, Valtonen ET (2006b) Susceptibility of eye flukeinfected fish to predation by bird hosts. Parasitology 132:575-579. doi: $10.1017 / \mathrm{S} 0031182005009431$

Seppälä O, Karvonen A, Valtonen ET (2008) Shoaling behaviour of fish under parasitism and predation risk. Anim Behav 75:145-150. doi: 10.1016/j.anbehav.2007.04.022

Seppälä O, Karvonen A, Valtonen ET (2011) Eye fluke-induced cataracts in natural fish populations: is there potential for host manipulation? Parasitology 138:209-214. doi:10.1017/S0031182010001228

Seppälä O, Karvonen A, Valtonen ET (2012) Behavioural mechanisms underlying 'specific' host manipulation by a trophically transmitted parasite. Evol Ecol Res 14:73-81

Shariff M, Richards RH, Sommerville C (1980) The histopathology of acute and chronic infections of rainbow trout Salmo gairdneri Richardson with eye flukes, Diplostomum spp. J Fish Dis 3:455465. doi:10.1111/j.1365-2761.1980.tb00432.x

Sih A, Bell A, Johnson JC (2004) Behavioral syndromes: an ecological and evolutionary overview. Trends Ecol Evol 19:372-378. doi:10. 1016/j.tree.2004.04.009

Simberloff D, Gibbons L (2004) Now you see them, now you don't!population crashes of established introduced species. Biol Invasions 6:161-172. doi:10.1023/B:BINV.0000022133.49752.46

Sweeting RA (1974) Investigations into natural and experimental infections of freshwater fish by the common eye-fluke Diplostomum spathaceum Rud. Parasitology 69:291-300

Thernau T (2013) Survival, a package for survival analysis in S. R package version 2:37 http://cran.r-project.org/web/packages/survival/ index.html

Voutilainen A, Figueiredo K, Huuskonen H (2008) Effects of the eye fluke Diplostomum spathaceum on the energetics and feeding of Arctic charr Salvelinus alpinus. J Fish Biol 73:2228-2237. doi:10. 1111/j.1095-8649.2008.02050.x

Wootten R (1974) Observations on strigeid metacercariae in the eyes of fish from Hanningfield reservoir, Essex, England. J Helminthol 48: 73-83. doi:10.1017/S0022149X00022628 